\title{
EMISSIONS DUE TO FOSSIL-FUEL CONSUMPTION AND CEMENT PRODUCTION IN TURKEY (1970-1991)
}

\author{
KATALIN KOVARI ZAIM \\ Institute of Environmental Science, Bogazici University, Bebek-80815, Istanbul, Turkey
}

(Received 3 November 1994)

\begin{abstract}
Standard emission factors are used for estimating levels of particulate matter (PM), $\mathrm{SO}_{x}, \mathrm{CO}$, volatile organic compounds (VOC), $\mathrm{NO}_{x}$ and $\mathrm{CO}_{2}$. Results are presented for different fuels and energy-consuming sectors. In the early 1970s households utilizing lignite made the most significant contributions to emissions, while manufacturing industries with both lignite and petroleum utilization were responsible for $\mathrm{SO}_{x}, \mathrm{NO}_{x}$, and PM emissions. Households continued to produce the same $\mathrm{CO}$ and VOC emissions and manufacturing industries continued to be responsible for $\mathrm{NO}_{x}$ emissions through the 1970-1990 period. Power production had gained in importance with regard to $\mathrm{SO}_{x}, \mathrm{CO}_{2}$, and $\mathrm{PM}$ emissions by 1990 .
\end{abstract}

\section{INTRODUCTION}

Air pollution is a symptom of industrial growth in developed and developing countries alike. ${ }^{1}$ Among the most critical compounds affecting the quality of our atmosphere are $\mathrm{SO}_{2}, \mathrm{NO}_{x}, \mathrm{CO}, \mathrm{O}_{3}$, and $\mathrm{CO}_{2}$. In the late $1980 \mathrm{~s}$, the international community responded to air pollution through environmental initiatives with the objective of establishing international emissions standards. The European community announced that emissions from large combustion plants ought to be reduced below the 1980 emissions level by $20 \%$ by the end of $1993,40 \%$ by 1998 and $60 \%$ by 2003 . Turkey is a developing country. There is very limited information available on annual pollutant emissions. In this study, we assess airpollution indicators in Turkey for the period 1970-1991, using yearly fossil-fuel consumption as basis for the computations.

\section{DATA AND METHODOLOGY}

Fossil-fuel consumption data have been collected from yearly publications on energy statistics in Turkey. ${ }^{2}$ The polluting sectors are households, manufacturing industry, power production, and transportation. Fossil-fuel consumptions includes the use of coal, lignite, petroleum, and natural gas (NG). For estimations of air pollution, methods suggested by the U.S. EPA ${ }^{3}$ and Marland ${ }^{4}$ were applied. The EPA method yields concentrations of $\mathrm{SO}_{2}, \mathrm{NO}_{x}, \mathrm{CO}$, particulate matter (PM), and volatile organic compounds (VOC) for fossil-fuels using standard emission factors.

The energy-consuming sectors of Turkey are identified in the manner of Tasdemiroglu. ${ }^{5}$ Standard tables for uncontrolled emissions are chosen for computations of yearly emissions of $\mathrm{SO}_{2}, \mathrm{NO}_{x}, \mathrm{CO}$, VOC, and PM for 1970-1991. Marland's method ${ }^{4}$ was applied to calculate $\mathrm{CO}_{2}$ emissions. Yearly $\mathrm{CO}_{2}$ emissions $\left(\mathrm{CO}_{2 i}\right)$ are determined by the amount of fuel consumed $\left(P_{i}\right)$, the fraction of fuel that is oxidized $\left(\mathrm{FO}_{i}\right)$ and the carbon content of the fuel $\left(C_{i}\right)$, i.e.

$$
\mathrm{CO}_{2 i}=\left(P_{i}\right)\left(\mathrm{FO}_{i}\right)\left(C_{i}\right) \text {, }
$$

where the subscript $i$ indicates a particular fuel group and $\mathrm{CO}_{2 i}$ is the mass of carbon. All coal, NG or crude oil are not of the same composition and hence have different $\mathrm{CO}_{2}$ potentials. Results were obtained according to Marland's method and are listed in Table 1.

The most significant contributors to $\mathrm{CO}_{2}$ emissions are fossil-fuel burning and cement manufacturing. Since cement production requires the use of both fossil-fuels and $\mathrm{CaCO}_{3}$, its $\mathrm{CO}_{2}$ emissions are significant. According to Marland, ${ }^{4}$ for each mole of $\mathrm{CaO}$ produced from $\mathrm{CaCO}_{3}$, one mole of $\mathrm{CO}_{2}$ is emitted. Thus, in the production of one metric ton of cement, $0.137 \mathrm{mt}$ of $\mathrm{C}$ is released into the atmosphere as $\mathrm{CO}_{2}$. 
Table 1. Factors and units for calculating annual $\mathrm{CO}_{2}$ emissions in $10^{6} \mathrm{mt}$ of $\mathrm{C}$ from Eq. (1).

NG consumption $\left(P_{\mathrm{g}}\right)=$ annual consumption $( \pm 10 \%)$ of $\mathrm{NG}$ in $Q\left(10^{15} \mathrm{~J}\right), \mathrm{FO}_{\mathrm{g}}=$ effective fraction oxidized in the year of consumption $=0.98 \pm 1 \%, C_{\mathrm{g}}=$ carbon content in $10^{6} \mathrm{mt}$ per $10^{15 \mathrm{~J}}=0.0137 \pm 2 \%$.

Petroleum and NG consumption $\left(\boldsymbol{P}_{1}\right)=$ annual consumption in $10^{6} \mathrm{mt}( \pm 8 \%), \mathrm{FO}_{1}=$ effective fraction oxidized in the year of consumption $=0.918 \pm 3 \%, C_{1}=$ carbon content in $\mathrm{mt} \mathrm{C}$ per $\mathrm{mt}$ crude oil $=0.85 \pm 1 \%$.

Coal consumption $\left(P_{\mathrm{S}}\right)=$ annual consumption in $10^{6} \mathrm{mt}$ coal equivalent $( \pm 11.2 \%), \mathrm{FO}_{\mathrm{S}}=$ effective fraction oxidized in the year of consumption $=0.982 \pm 2 \%, C_{\mathrm{S}}=$ carbon content in $\mathrm{mt} \mathrm{C}$ per $\mathrm{mt}$ coal equivalent $=0.746 \pm 2 \%$.

\section{RESULTS AND DISCUSSION}

For each of the pollutants, the contribution made by fossil-fuel consumption has been computed (see Fig. 1). Prior to 1979, the major contributor to $\mathrm{CO}_{2}$ was petroleum consumption; after 1980, lignite consumption became dominant. The importance of lignite as a major contributing factor in the production of $\mathrm{SO}_{2}, \mathrm{PM}$, and $\mathrm{CO}$ may be observed in Figs. 2-5.

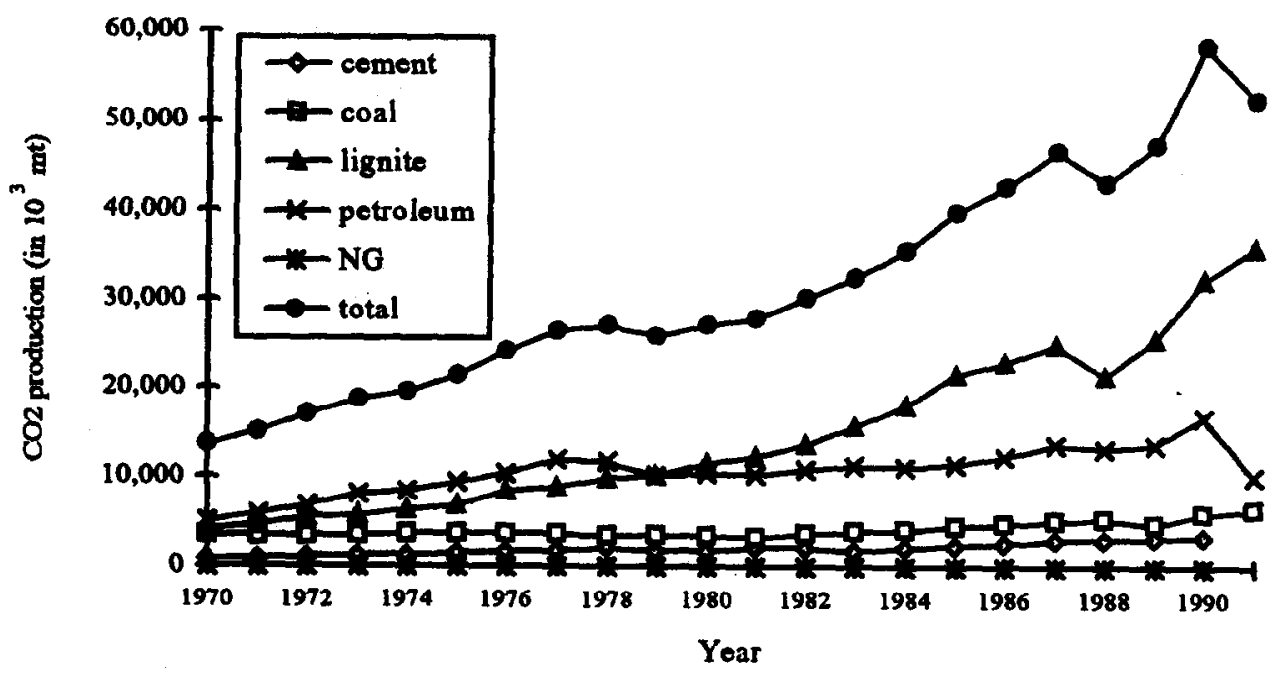

Fig. 1. Annual $\mathrm{CO}_{2}$ production in Turkey from fossil-fuel burning (1970-1991).

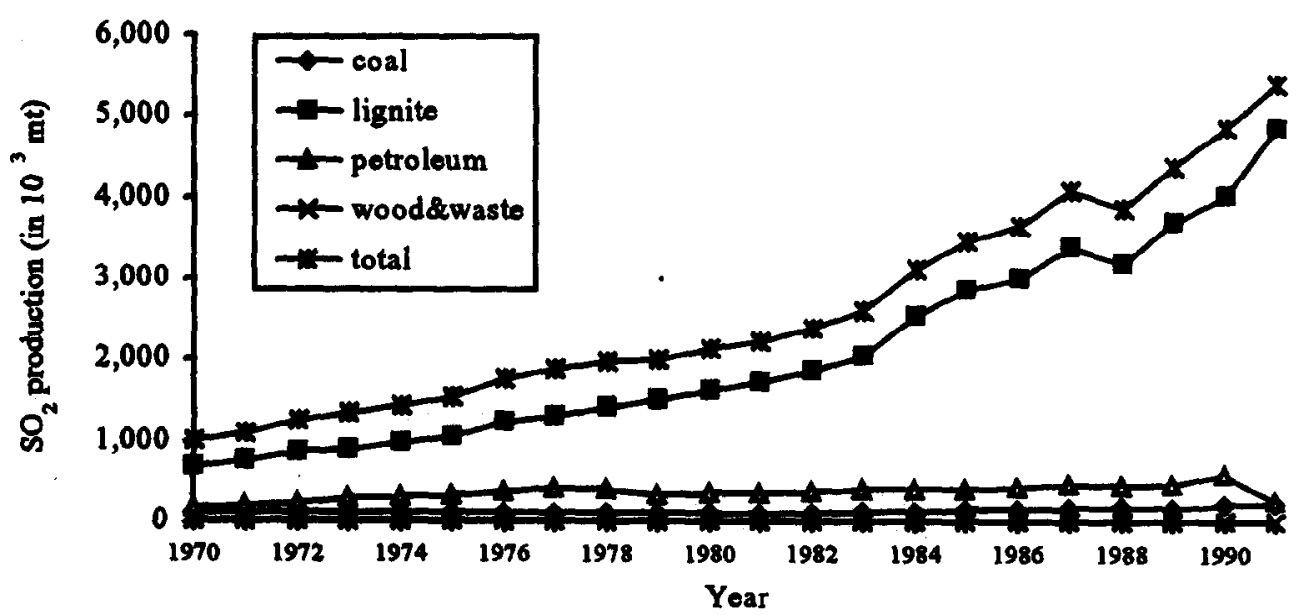

Fig. 2. Annual $\mathrm{SO}_{2}$ production in Turkey from fossil-fuel burning (1970-1991). 


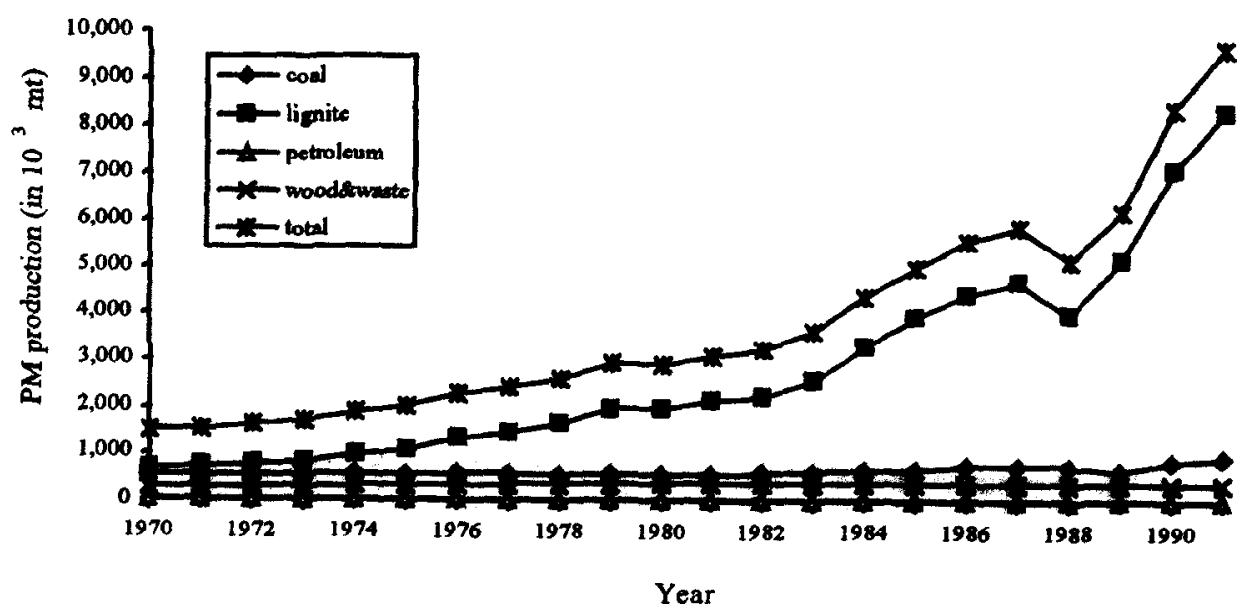

Fig. 3. Annual PM production in Turkey from fossil-fuel burning (1970-1991).

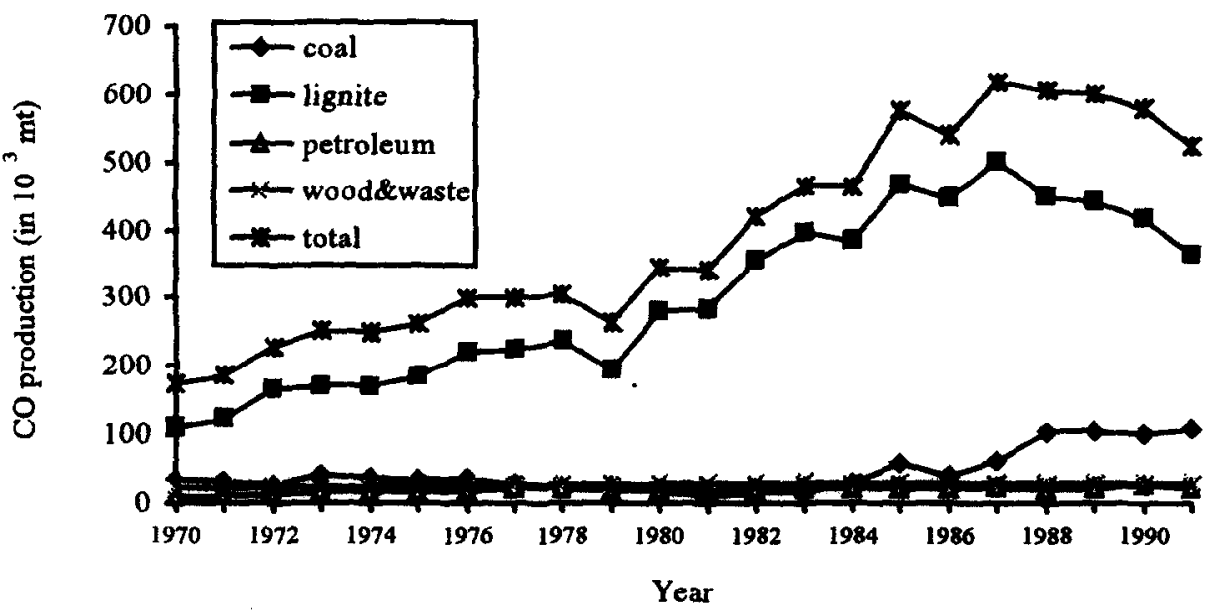

Fig. 4. Annual CO production in Turkey from fossil-fuel burning (1970-1991).

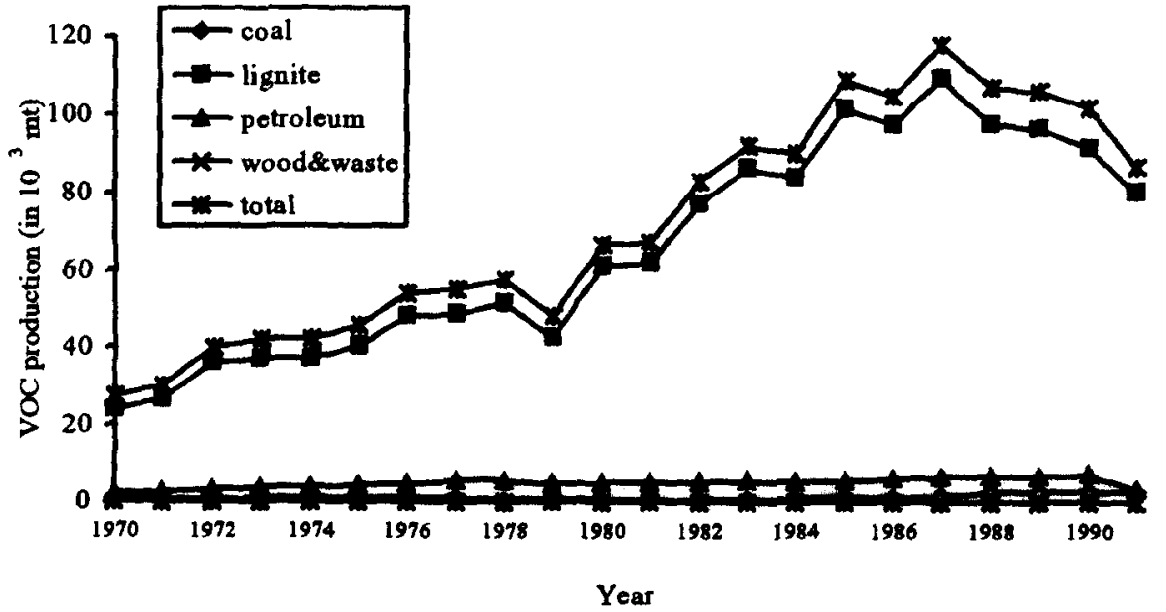

Fig. 5. Annual VOC production in Turkey from fossil-fuel burning (1970-1991). 


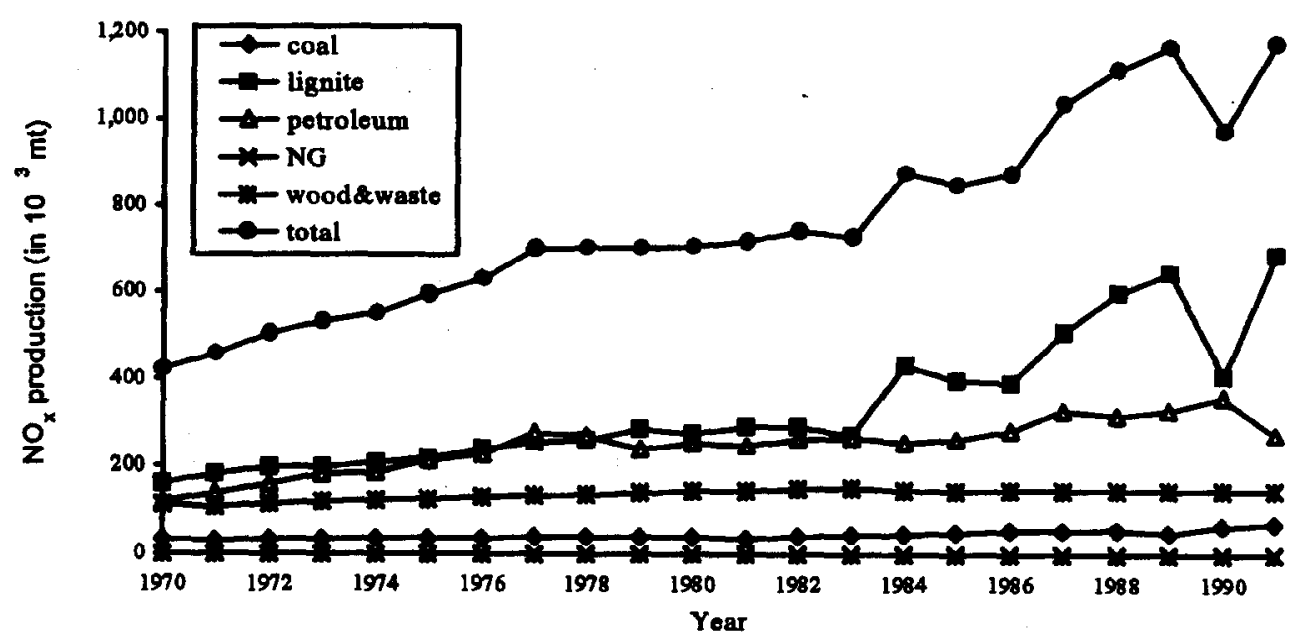

Fig. 6. Annual $\mathrm{NO}_{x}$ production in Turkey from fossil-fuel burning (1970-1991).

For $\mathrm{NO}_{x}$ production, both lignite and petroleum consumption play important roles (see Fig. 6).

$\mathrm{SO}_{2}$ production for various sectors is summarized in Table 2 for various fossil-fuels, wood and waste in five-year intervals from 1970-1990. Similar tables are given for $\mathrm{NO}_{x}, \mathrm{CO}_{2}, \mathrm{CO}$, VOC, and PM in Tables 3-7, respectively. These tables show which combination of sectors and fossil-fuel consumption is most detrimental for a specific air-pollution indicator. Furthermore, we see how the contributions change over time.

Industrial activities using lignite were responsible for $41 \%$ of emissions in 1970 for $\mathrm{SO}_{2}$. Their importance has decreased with time and reached a level of $21 \%$ in 1990 . At the same time, power production using lignite as an energy source increased. In 1990, power production was responsible for $53 \%$ of $\mathrm{SO}_{2}$ emissions.

For $\mathrm{NO}_{x}$ emissions, contributed $56 \%$ in 1970 and $51 \%$ in 1990 . The decreasing importance of industrial activities for $\mathrm{NO}_{x}$ emissions was more than offset by the increased importance of power production (see Table 3).

Household lignite consumption contributed $42 \%$ of $\mathrm{CO}_{2}$ emissions 1970 and then decreased to $20 \%$ by 1990 . Power production using lignite gained in importance and contributed $37.8 \%$ by 1990 . Cement production was responsible for about $6 \%$ of $\mathrm{CO}_{2}$ emissions between 1970 and 1990.

Table 2. Sectoral shares of $\mathrm{SO}_{2}$ emissions (in \%); subtotals add to $100 \%$.

\begin{tabular}{|c|c|c|c|c|c|}
\hline Source & 1970 & 1975 & 1980 & 1985 & 1990 \\
\hline $\begin{array}{l}\text { Household - Coal } \\
\text { - Lignite } \\
\text { - } \\
\text { - Petroleum } \\
\text { Subtotal }\end{array}$ & $\begin{array}{r}1.69 \\
18.64 \\
2.80 \\
1.53 \\
24.66\end{array}$ & $\begin{array}{c}1.13 \\
20.21 \\
3.3 \\
1.13 \\
25.77\end{array}$ & $\begin{array}{r}0.39 \\
22.15 \\
2.52 \\
0.94 \\
26\end{array}$ & $\begin{array}{r}0.88 \\
22.58 \\
1.46 \\
0.54 \\
25.46\end{array}$ & $\begin{array}{c}1.11 \\
14.1 \\
2.38 \\
0.42 \\
18.01\end{array}$ \\
\hline $\begin{array}{l}\text { Industry } \\
\text { - Coal } \\
\text { - Lignite } \\
\text {-Petroleum } \\
\text { Subtotal }\end{array}$ & $\begin{array}{r}1.05 \\
40.23 \\
0.43 \\
41.71\end{array}$ & $\begin{array}{r}1.10 \\
36.24 \\
0.49 \\
37.84\end{array}$ & $\begin{array}{r}0.66 \\
32.79 \\
0.43 \\
33.88\end{array}$ & $\begin{array}{r}0.49 \\
28.99 \\
0.26 \\
29.74\end{array}$ & $\begin{array}{r}0.70 \\
21.38 \\
0.26 \\
22.34\end{array}$ \\
\hline $\begin{array}{l}\text { Power production-Coal } \\
\qquad \begin{array}{l}\text { - Lignite } \\
\text {-Petroleum }\end{array} \\
\text { Subtotal }\end{array}$ & $\begin{array}{r}7.41 \\
9.09 \\
4.53 \\
21.84\end{array}$ & $\begin{array}{r}5.06 \\
12.17 \\
6.64 \\
23.87\end{array}$ & $\begin{array}{r}4.11 \\
21.57 \\
5.22 \\
30.9\end{array}$ & $\begin{array}{r}3.18 \\
31.03 \\
3.81 \\
38.02\end{array}$ & $\begin{array}{r}2.76 \\
47.26 \\
3.16 \\
53.18\end{array}$ \\
\hline $\begin{array}{l}\text { Transportation-Coal } \\
\text { Subtotal } \quad \text {-Petroleum }\end{array}$ & $\begin{array}{l}1.89 \\
9.9 \\
11.79\end{array}$ & $\begin{array}{r}0.96 \\
11.56 \\
12.52\end{array}$ & $\begin{array}{l}0.26 \\
8.96 \\
9.22\end{array}$ & $\begin{array}{l}0.19 \\
6.59 \\
6.78\end{array}$ & $\begin{array}{l}0.05 \\
6.42 \\
6.47\end{array}$ \\
\hline
\end{tabular}


Table 3. Sectoral shares of $\mathrm{NO}_{x}$ emissions (in \%); subtotals add to $100 \%$.

\begin{tabular}{|c|c|c|c|c|c|}
\hline Source & 1970 & 1975 & 1980 & 1985 & 1990 \\
\hline Household_-Coal & 0.25 & 0.18 & 0.07 & 0.20 & 0.28 \\
\hline - Lignite & 0.84 & 0.98 & 1.21 & 1.56 & 1.07 \\
\hline -Petroleum & 0.28 & 0.35 & 0.3 & 0.22 & 0.4 \\
\hline -Natural gas & 0.01 & 0.01 & 0.00 & 0.00 & 0.00 \\
\hline -Wood and waste & 25.41 & 20.31 & 18.82 & 14.56 & 11.63 \\
\hline Subtotal & 26.79 & 21.83 & 20.4 & 16.54 & 13.38 \\
\hline Industry-Coal & 0.88 & 0.99 & 0.67 & 0.63 & 0.98 \\
\hline -Lignite & 36.10 & 34.48 & 34.89 & 39.09 & 31.60 \\
\hline -Petroleum & 19.02 & 22.94 & 22.71 & 17.31 & 19.18 \\
\hline Subtotal & 56 & 58.41 & 58.27 & 57.07 & 51.76 \\
\hline Power production-Coal & 6.24 & 4.59 & 4.18 & 3.18 & 3.89 \\
\hline -Lignite & 2.49 & 3.61 & 7.15 & 31.03 & 21.78 \\
\hline -Petroleum & 3.46 & 5.47 & 4.8 & 3.81 & 4.04 \\
\hline Subtotal & 12.19 & 13.67 & 16.13 & 38.02 & 29.71 \\
\hline Transportation-Coal & 0.34 & 0.22 & 0.12 & 0.19 & 0.06 \\
\hline -Petroleum & 4.68 & 5.87 & 5.08 & 6.59 & 5.09 \\
\hline Subtotal & 5.02 & 6.09 & 5.2 & 6.78 & 5.15 \\
\hline
\end{tabular}

Table 4. Sectoral shares of $\mathrm{CO}_{2}$ emissions (in \%); subtotals add to $100 \%$.

\begin{tabular}{|c|c|c|c|c|c|}
\hline Source & 1970 & 1975 & 1980 & 1985 & 1990 \\
\hline Cement production & 6.36 & 6.93 & 6.49 & 6.05 & 5.72 \\
\hline Household-Coal & 5.41 & 5.15 & 4.25 & 4.02 & 5.06 \\
\hline -Lignite & 40.40 & 40.41 & 39.28 & 34.15 & 20.06 \\
\hline -Petroleum & 4.70 & 4.74 & 5.42 & 4.14 & 5.4330 .55 \\
\hline Subtotal & 51.51 & 50.30 & 48.95 & 42.31 & \\
\hline Industry-Coal & 2.35 & 2.39 & 1.58 & 1.30 & 1.05 \\
\hline -Lignite & 9.00 & 9.54 & 9.4 & 8.22 & 6.79 \\
\hline -Petroleum & 8.51 & 8.51 & 9.09 & 7.00 & 6.13 \\
\hline Subtotal & 19.86 & 20.44 & 20.07 & 16.52 & 13.97 \\
\hline Power production-Coal & 8.43 & 7.98 & 6.73 & 6.34 & 5.20 \\
\hline -Lignite & 4.44 & 4.46 & 11.32 & 20.04 & 37.00 \\
\hline -Petroleum & 3.77 & 4.29 & 3.09 & 3.04 & 3.04 \\
\hline Subtotal & 16.64 & 16.73 & 21.14 & 29.42 & 45.24 \\
\hline Transportation-Coal & 0.41 & 0.21 & 0.25 & 0.22 & 0.01 \\
\hline -Lignite & 0.51 & 0.07 & 0.03 & 0.09 & 0.01 \\
\hline -Petroleum & 4.71 & 5.32 & 3.31 & 5.29 & 4.5 \\
\hline Subtotal & 4.71 & 5.60 & 3.63 & 5.70 & 4.52 \\
\hline
\end{tabular}

$\mathrm{CO}$ emission is mostly related to household activities due to lignite burning which was responsible for about $80 \%$ of emissions in 1970 and $90 \%$ in 1990 (see Table 5). A similar situation applies for volatile organic compound ( $\mathrm{VOC}$ ) emissions. The household sector utilizing lignite and wood waste emitted $51.67 \%$ of the VOC in 1970 and $87.5 \%$ in 1990 (see Table 6).

Particulate matter (PM) emissions are summarized in Table 7. In 1970, power production and the manufacturing industry played significant roles with shares of $\mathbf{4 0}$ and $30 \%$, respectively. Power production utilizing coal was responsible for $25 \%$ of emissions in 1970 and $8 \%$ in 1990 . This reduction in PM emissions has been offset by the increased importance of lignite utilization in the same sector. 
Table 5. Sectoral shares of CO emissions (in \%); subtotals add to $100 \%$.

\begin{tabular}{|c|c|c|c|c|c|}
\hline Source & 1970 & 1975 & 1980 & 1985 & 1990 \\
\hline $\begin{array}{l}\text { Household-Coal } \\
\qquad \begin{array}{l}\text { - Lignite } \\
\text {-Petroleum }\end{array} \\
\text { Subtotal }\end{array}$ & $\begin{array}{r}18.03 \\
62.72 \\
1.17 \\
81.92\end{array}$ & $\begin{array}{r}12.39 \\
69.81 \\
0.91 \\
82.11\end{array}$ & $\begin{array}{r}4.52 \\
79.85 \\
0.84 \\
85.21\end{array}$ & $\begin{array}{r}4.82 \\
79.44 \\
0.73 \\
84.99\end{array}$ & $\begin{array}{r}11.28 \\
69.33 \\
1.40 \\
82.01\end{array}$ \\
\hline $\begin{array}{l}\text { Industry } \\
\text { - Coal } \\
\text { - Lignite } \\
\text {-Petroleum } \\
\text { Subtotal }\end{array}$ & $\begin{array}{l}0.12 \\
0.57 \\
3.44 \\
4.13\end{array}$ & $\begin{array}{l}0.13 \\
0.52 \\
2.53 \\
3.18\end{array}$ & $\begin{array}{l}0.08 \\
0.49 \\
2.74 \\
3.31\end{array}$ & $\begin{array}{l}0.06 \\
0.42 \\
2.45 \\
2.93\end{array}$ & $\begin{array}{l}0.11 \\
0.44 \\
2.9 \\
3.45\end{array}$ \\
\hline $\begin{array}{l}\text { Power production - Coal } \\
\text { - Lignite } \\
\text { - Petroleum } \\
\text { Subtotal }\end{array}$ & $\begin{array}{r}0.84 \\
1.46 \\
11.65 \\
13.95\end{array}$ & $\begin{array}{r}0.59 \\
2.87 \\
11.25 \\
14.71\end{array}$ & $\begin{array}{r}0.50 \\
0.20 \\
10.78 \\
11.48\end{array}$ & $\begin{array}{l}0.37 \\
1.22 \\
10.5 \\
12.08\end{array}$ & $\begin{array}{c}0.45 \\
2.69 \\
11.4 \\
14.54\end{array}$ \\
\hline
\end{tabular}

Table 6. Sectoral shares of VOC emissions (in \%); subtotals add to $100 \%$.

\begin{tabular}{|c|c|c|c|c|c|}
\hline Source & 1970 & 1975 & 1980 & 1985 & 1990 \\
\hline Household_Coal & 1.58 & 1.13 & 0.40 & 1.02 & 1.88 \\
\hline - Lignite & 47.53 & 55.38 & 60.25 & 71.71 & 65.64 \\
\hline -Petroleum & 0.63 & 0.80 & 0.63 & 0.41 & 0.98 \\
\hline -Wood and waste & 44.14 & 35.13 & 29.17 & 20.59 & 21.88 \\
\hline Subtotal & 93.88 & 92.44 & 90.45 & 93.73 & 90.38 \\
\hline Industry-Coal & 0.08 & 0.09 & 0.06 & 0.05 & 0.10 \\
\hline -Lignite & 0.63 & 0.6 & 0.55 & 0.56 & 0.60 \\
\hline -Petroleum & 1.94 & 2.33 & 2.11 & 1.44 & 2.12 \\
\hline Subtotal & 2.65 & 3.02 & 2.72 & 2.05 & 2.82 \\
\hline Power production-Coal & 0.10 & 0.08 & 0.06 & 0.10 & 0.07 \\
\hline -Lignite & 0.36 & 0.57 & 0.95 & 1.54 & 3.43 \\
\hline -Petroleum & 0.65 & 0.94 & 0.76 & 0.62 & 0.76 \\
\hline Subtotal & 1.11 & 1.59 & 4.49 & 2.26 & 4.26 \\
\hline Transportation-Petroleum & 2.36 & 2.95 & 2.34 & 1.96 & 2.54 \\
\hline
\end{tabular}

Table 7. Sectoral shares of PM emissions (in \%); subtotals add to $100 \%$.

\begin{tabular}{|c|c|c|c|c|c|}
\hline Source & 1970 & 1975 & 1980 & 1985 & 1990 \\
\hline Household-Coal & 0.07 & 0,05 & 0.01 & 0.04 & 0.04 \\
\hline -Lignite & 1.61 & 2.02 & 2.1 & 2.03 & 1.05 \\
\hline -Petroleum & 0.06 & 0.08 & 0.06 & 0.03 & 0.04 \\
\hline -Wood and waste & 18.98 & 16.25 & 12.78 & 7.40 & 4.48 \\
\hline Subtotal & 20.72 & 18.40 & 14.95 & 9.50 & 5.61 \\
\hline Industry - Coal & 3.59 & 4.32 & 2.49 & 1.75 & 2.06 \\
\hline —Lignite & 27.29 & 27.91 & 24.00 & 20.12 & 12.33 \\
\hline -Petroleum & 0.29 & 0.37 & 0.31 & 0.18 & 0.15 \\
\hline Subtotal & 31.17 & 32.60 & 26.80 & 22.05 & 14.54 \\
\hline $\begin{array}{c}\text { Power production —Coal } \\
\text { _Lignite }\end{array}$ & 25.14 & 19.83 & 15.34 & 11.23 & $\begin{array}{r}8.10 \\
7140\end{array}$ \\
\hline - - Petroleum & $\begin{array}{r}15.80 \\
0.07\end{array}$ & $\begin{array}{r}24.55 \\
0.13\end{array}$ & $\begin{array}{r}41.35 \\
0.10\end{array}$ & $\begin{array}{r}30.4 \\
0.06\end{array}$ & $\begin{array}{r}11.40 \\
0.04\end{array}$ \\
\hline Subtotal & 41.07 & 44.51 & 56.79 & 67.69 & 79.54 \\
\hline Transportation-Coal & 6.57 & 3.87 & 1.07 & 0.49 & 0.09 \\
\hline -Petroleum & 0.47 & 0.62 & 0.39 & 0.27 & 0.22 \\
\hline Subtotal & 7.04 & 4.49 & 1.46 & 0.76 & 0.31 \\
\hline
\end{tabular}




\section{REFERENCES}

1. National Research Council, "Changing Climate: Report of the Carbon Dioxide Assessment Committee", National Academy Press, Washington, DC (1983).

2. Ministry of Energy and Natural Resources, "Energy Report 1970-1992" (in Turkish), Ankara, Turkey (1993).

3. U.S. Environmental Protection Agency, "Compilation of Air Pollution Emission Factors", Vols. 1 and 2, 4th edn., Publ. No. A3-42, Research Triangle Park, NC (September 1985).

4. G. Marland and R. M. Rotty, Tellus 36B, 232 (1984).

5. E. Tasdemiroglu, Energy-The Intermational Journal 17, 95 (1992). 